\title{
Projective maximal families of orthogonal measures with large continuum
}

\author{
VERA FISCHER \\ SY DAVID FRIEDMAN \\ ASGER TÖRNQUIST
}

\begin{abstract}
We study maximal orthogonal families of Borel probability measures on $2^{\omega}$ (abbreviated m.o. families) and show that there are generic extensions of the constructible universe $L$ in which each of the following holds:

(1) There is a $\Delta_{3}^{1}$-definable well-ordering of the reals, there is a $\Pi_{2}^{1}$-definable m.o. family, there are no $\boldsymbol{\Sigma}_{2}^{1}$-definable m.o. families and $\mathfrak{b}=\mathfrak{c}=\omega_{3}$ (in fact any reasonable value of $\mathfrak{c}$ will do).

(2) There is a $\Delta_{3}^{1}$-definable well-ordering of the reals, there is a $\Pi_{2}^{1}$-definable m.o. family, there are no $\Sigma_{2}^{1}$-definable m.o. families, $\mathfrak{d}=\omega_{1}$ and $\mathfrak{c}=\omega_{2}$.

2010 Mathematics Subject Classification 03E15, 03E17, 03E20, 03E35, 03 E45 (primary)
\end{abstract}

Keywords: projective families of orthogonal measures, projective wellorders, large continuum

\section{Introduction}

Let $X$ be a Polish space, and let $P(X)$ denote the Polish space of Borel probability measures on $X$, in the sense of $[9,17 . E]$. Recall that if $\mu, \nu \in P(X)$ then $\mu$ and $\nu$ are said to be orthogonal, written $\mu \perp \nu$, if there is a Borel set $B \subseteq X$ such that $\mu(B)=0$ and $\nu(X \backslash B)=0$. A set of measures $\mathcal{A} \subseteq P(X)$ is said to be orthogonal if whenever $\mu, \nu \in \mathcal{A}$ and $\mu \neq \nu$ then $\mu \perp \nu$. A maximal orthogonal family, or m.o. family, is an orthogonal family $\mathcal{A} \subseteq P(X)$ which is maximal under inclusion.

The present paper is concerned with the study of definable m.o. families. A well-known result due to Preiss and Rataj [13] states that there are no analytic m.o. families. In a recent paper [3] it was shown by Fischer and Törnquist that if all reals are constructible then there is a $\Pi_{1}^{1}$ m.o. family. The latter paper also raised the question how restrictive the existence of a definable m.o. family is on the structure of the real line, since it was shown that $\Pi_{1}^{1}$ m.o. families cannot coexist with Cohen reals.

In the present paper we study $\Pi_{2}^{1}$ m.o. families in the context of $\mathfrak{c} \geq \omega_{2}$, with the additional requirement that there is a $\Delta_{3}^{1}$-definable wellorder of $\mathbb{R}$. Our main results are: 
Theorem 1 It is consistent with $\mathfrak{c}=\mathfrak{b}=\omega_{3}$ that there is a $\Delta_{3}^{1}$-definable wellorder of the reals, a $\Pi_{2}^{1}$ definable maximal orthogonal family of measures and there are no $\Sigma_{\mathbf{2}}^{\mathbf{1}}$-definable maximal sets of orthogonal measures.

There is nothing special about $\mathfrak{c}=\omega_{3}$ : The same result can be obtained for any reasonable value of $\mathfrak{c}$.

Theorem 2 It is consistent with $\mathfrak{d}=\omega_{1}, \mathfrak{c}=\omega_{2}$ that there is a $\Delta_{3}^{1}$-definable wellorder of the reals, a $\Pi_{2}^{1}$ definable maximal orthogonal family of measures and there are no $\boldsymbol{\Sigma}_{\mathbf{2}}^{\mathbf{1}}$-definable maximal sets of orthogonal measures.

Taken together these theorems indicate that the existence of a $\Pi_{2}^{1}$ m.o. family does not seem to impose any severe restrictions on the structure of the real line. On the other hand, we show (Proposition 1) that $\boldsymbol{\Sigma}_{\mathbf{2}}^{\mathbf{1}}$ m.o. families cannot coexist with either Cohen or random reals, extending the previous result of Fischer and Törnquist that $\Pi_{1}^{1}$ m.o. families cannot coexist with Cohen reals. This is the explanation why in the models produced to prove Theorems 1 and 2 there are no $\Sigma_{2}^{1}$ m.o. families.

The theorems of this paper belong to a line of results concerning the definability of certain combinatorial objects on the real line and in particular the question of how low in the projective hierarchy such objects exist. In [12] Mathias showed that there is no $\boldsymbol{\Sigma}_{1}^{1}$-definable maximal almost disjoint (mad) family in $[\omega]^{\omega}$. Assuming $V=L$, Miller obtained a $\Pi_{1}^{1} \operatorname{mad}$ family in $[\omega]^{\omega}$, see [11].

The study of the existence of definable combinatorial objects on $\mathbb{R}$ in the presence of a projective wellorder of the reals and $\mathfrak{c} \geq \omega_{2}$ was initiated in [1], [4] and [2]. The wellorder of $\mathbb{R}$ in all those models has a $\Delta_{3}^{1}$-definition, which is indeed optimal for models of $\mathfrak{c} \geq \omega_{2}$, since by Mansfield's theorem (see [7, Theorem 25.39]) the existence of a $\Sigma_{2}^{1}$-definable wellorder of the reals implies that all reals are constructible. The existence of a $\Pi_{2}^{1}$-definable $\omega$-mad family in $[\omega]^{\omega}$ in the presence of $\mathfrak{c}=\mathfrak{b}=\omega_{2}$ was established by Friedman and Zdomskyy in [4]. In the same paper, referring to earlier results (see [14] and [8]) they outlined the construction of a model in which $\mathfrak{c}=\omega_{2}$ and there is a $\Pi_{1}^{1}$-definable $\omega$-mad family: Start with the constructible universe $L$, obtain a $\Pi_{1}^{1}$-definable $\omega$ mad family and proceed with a countable support iteration of length $\omega_{2}$ of Miller forcing. The techniques were further developed in [2] to establish a model in which there is a $\Pi_{2}^{1}$-definable $\omega$-mad family and $\mathfrak{c}=\mathfrak{b}=\omega_{3}$. In particular, in the models from [4] and [2], there are no maximal almost disjoint families of size $<\mathfrak{c}$ and so the almost disjointness number has a $\Pi_{2}^{1}$-witness.

The present paper combines the encoding techniques of [3] with the techniques of $[1,4,2]$ to obtain Theorems 1 and 2. We note that one significant difference from the situation for mad families is that m.o. families always have size $\mathfrak{c}$ (see [3, Proposition 4.1]). Moreover, owing to the fact that our coding technique for measures (Lemma 1) preserves the measure class, the forcing constructions in $\S 3$ and $\S 4$ is somewhat simplified compared to $[1,4,2]$.

Acknowledgement. The authors would like to thank the Austrian Science Fund FWF for generous support through grants no. P 20835-N13 (Fischer, Friedman), and P 19375-N18 (Friedman, Törnquist), as well as a Marie Curie grant from the European Union no. IRG-249167 (Törnquist). The paper was written while Asger Törnquist was a post-doctoral fellow at the Kurt Gödel Research Center. 


\section{Preliminaries}

In this section, we briefly recall the coding of probability measures on $2^{\omega}$ and the encoding technique for measures introduced in [3].

Let $X$ be a Polish space. Recall that if $\mu, \nu \in P(X)$ then $\mu$ is said to be absolutely continuous with respect to $\nu$, written $\mu \ll \nu$, if for all Borel subsets of $X$ we have that $\nu(B)=0$ implies that $\mu(B)=0$. Two measures $\mu, \nu \in P\left(2^{\omega}\right)$ are called absolutely equivalent, written $\mu \approx \nu$, if $\mu \ll \nu$ and $\nu \ll \mu$.

If $s \in 2^{<\omega}$ we let $N_{s}=\left\{x \in 2^{\omega}: s \subseteq x\right\}$ be the basic neighbourhood determined by $s$. Following [3], we let

$$
p\left(2^{\omega}\right)=\left\{f: 2^{<\omega} \rightarrow[0,1]: f(\emptyset)=1 \wedge\left(\forall s \in 2^{<\omega}\right) f(s)=f\left(s^{\curlyvee} 0\right)+f\left(s^{\frown} 1\right)\right\} .
$$

The spaces $p\left(2^{\omega}\right)$ and $P\left(2^{\omega}\right)$ are homeomorphic via the recursive isomorphism $f \mapsto \mu_{f}$ where $\mu_{f} \in P\left(2^{\omega}\right)$ is the measure uniquely determined by requiring that $\mu_{f}\left(N_{s}\right)=f(s)$ for all $s \in 2^{<\omega}$. We call the unique real $f \in p\left(2^{\omega}\right)$ such that $\mu=\mu_{f}$ the code for $\mu$. The identification of $P\left(2^{\omega}\right)$ and $p\left(2^{\omega}\right)$ allows us to use the notions of effective descriptive set theory in the space $P\left(2^{\omega}\right)$. For instance, the set $P_{c}\left(2^{\omega}\right)$ of all non-atomic probability measures on $2^{\omega}$ is arithmetical because the set $p_{c}\left(2^{\omega}\right)=\left\{f \in p\left(2^{\omega}\right): \mu_{f}\right.$ is non-atomic $\}$ is easily seen to be arithmetical, as shown in [3].

We will use the method of coding a real $z \in 2^{\omega}$ into a measure $\mu \in P_{c}\left(2^{\omega}\right)$ introduced in [3]. For convenience we recall the construction in minimal detail. Given $\mu \in P_{c}\left(2^{\omega}\right)$ and $s \in 2^{<\omega}$ we let $t(s, \mu)$ be the lexicographically least $t \in 2^{<\omega}$ such that $s \subseteq t, \mu\left(N_{t \frown 0}\right)>0$ and $\mu\left(N_{t^{\wedge} 1}\right)>0$, if it exists and otherwise we let $t(s, \mu)=\emptyset$. Define recursively $t_{n}^{\mu} \in 2^{<\omega}$ by letting $t_{0}^{\mu}=\emptyset$ and $t_{n+1}^{\mu}=t\left(t_{n}^{\mu \frown} 0, \mu\right)$. Since $\mu$ is non-atomic, we have $\operatorname{lh}\left(t_{n+1}^{\mu}\right)>\operatorname{lh}\left(t_{n}^{\mu}\right)$. Let $t_{\infty}^{\mu}=\bigcup_{n=0}^{\infty} t_{n}^{\mu}$. For $f \in p_{c}\left(2^{\omega}\right)$ and $n \in \omega \cup\{\infty\}$ we will write $t_{n}^{f}$ for $t_{n}^{\mu_{f}}$. Clearly the sequence $\left(t_{n}^{f}: n \in \omega\right)$ is recursive in $f$.

Define the relation $R \subseteq p_{c}\left(2^{\omega}\right) \times 2^{\omega}$ as follows:

$$
\begin{aligned}
R(f, z) \Longleftrightarrow(\forall n \in \omega)(z(n) & \left.=1 \longleftrightarrow\left(f\left(t_{n}^{f\urcorner} 0\right)=\frac{2}{3} f\left(t_{n}^{f}\right) \wedge f\left(t_{n}^{f} 1\right)=\frac{1}{3} f\left(t_{n}\right)\right)\right) \\
\wedge(z(n) & \left.=0 \leftrightarrow f\left(t_{n}^{f\urcorner} 0\right)=\frac{1}{3} f\left(t_{n}^{f}\right) \wedge f\left(t_{n}^{f} 1\right)=\frac{2}{3} f\left(t_{n}^{f}\right)\right) .
\end{aligned}
$$

Whenever $(f, z) \in R$ we say that $f$ codes $z$. Note that $\operatorname{dom}(R)=\left\{f \in p_{c}\left(2^{\omega}\right):(\exists z) R(f, z)\right\}$ is $\Pi_{1}^{0}$ and so the function $r: \operatorname{dom}(R) \rightarrow 2^{\omega}$, where $r(f)=z$ if and only if $(f, z) \in R$, is also $\Pi_{1}^{0}$. The key properties of this construction is contained in the following Lemma (see [3, Coding Lemma]):

Lemma 1 There is a recursive function $\bar{r}: p_{c}\left(2^{\omega}\right) \times 2^{\omega} \rightarrow p_{c}\left(2^{\omega}\right)$ such that $\mu_{\bar{r}(f, z)} \approx \mu_{f}$ and $R(\bar{r}(f, z), z)$ for all $f \in p_{c}\left(2^{\omega}\right)$ and $z \in 2^{\omega}$.

The proofs of Theorems 1 and 2 use the following result, which we now prove. 
Proposition 1 Let $a \in \mathbb{R}$ and suppose that there either is a Cohen real over $L[a]$ or there is a random real over $L[a]$. Then there is no $\Sigma_{2}^{1}(a)$ m.o. family.

We first need a preparatory Lemma. In $2^{\omega}$, consider the equivalence $E_{I}$ defined by

$$
x E_{I} y \Longleftrightarrow \sum_{n=0}^{\infty} \frac{|x(n)-y(n)|}{n+1}<\infty .
$$

We identify $2^{\omega}$ with $\mathbb{Z}_{2}^{\omega}$ and equip it with the Haar measure $\mu$.

Lemma 2 Let $A \subseteq 2^{\omega}$ be a Borel set such that $\mu(A)>0$. Then $E_{I} \leq_{B} E_{I} \uparrow A$, where $E_{I}\lceil A$ is the restriction of $E_{I}$ to A.

Notation: The constant 0 sequence of length $n \in \omega \cup\{\infty\}$ is denoted $0^{n}$. If $A \subseteq 2^{\omega}$ and $s \in 2^{<\omega}$ let

$$
A_{(s)}=\left\{x \in 2^{\omega}: s \frown x \in A\right\},
$$

the localization of $A$ at $s$.

Proof of Lemma 2 We may assume that $A \subseteq 2^{\omega}$ is closed. We will define $q_{n} \in \omega, s_{n, i}, s_{t} \in 2^{<\omega}$ recursively for all $n \in \omega, i \in\{0,1\}$ and $t \in 2^{<\omega}$ satisfying

(1) $q_{0}=0$ and $q_{n+1}=q_{n}+\operatorname{lh}\left(s_{n, 0}\right)$.

(2) $s_{0, i}=\emptyset$ and $\operatorname{lh}\left(s_{n, i}\right)=\operatorname{lh}\left(s_{n, 1-i}\right)>0$ when $n>0$.

(3) $s_{\emptyset}=\emptyset$ and $s_{t} \frown i=s_{t} \frown s_{\operatorname{lh}(t)+1, i}$ for all $t \in 2^{<\omega}, i \in\{0,1\}$.

(4) $\frac{1}{n+1} \leq \sum_{k=0}^{\operatorname{lh}\left(s_{n+1,0}\right)} \frac{\left|s_{n+1,0}(k)-s_{n+1,1}(k)\right|}{q_{n}+k+1} \leq \frac{2}{n+1}$.

(5) $N_{s_{t}} \subseteq A$.

(6) If $t \in 2^{n}$ then $\mu\left(A_{\left(s_{t}\right)}\right)>1-2^{-n}$.

Suppose this can be done. We claim that the map $2^{\omega} \rightarrow A: x \mapsto a_{x}$ defined by

$$
a_{x}=\bigcup_{n \in \omega} s_{x \uparrow n}
$$

is a Borel (in fact, continuous) reduction of $E_{I}$ to $E_{I}\left\lceil A\right.$. To see this, fix $x, y \in 2^{\omega}$ and note that by (4) we have that

$$
\sum_{n=0}^{\infty} \frac{|x(n)-y(n)|}{n+1} \leq \sum_{n=0}^{\infty} \sum_{k=0}^{\operatorname{lh}\left(s_{n+1,0}\right)} \frac{\left|s_{n+1, x(i)}(k)-s_{n+1, y(i)}(k)\right|}{q_{n}+k+1}=\sum_{n=0}^{\infty} \frac{\left|a_{x}(n)-a_{y}(n)\right|}{n+1} \leq 2 \sum_{n=0}^{\infty} \frac{|x(n)-y(n)|}{n+1}
$$

so that $x E_{I} y$ if and only if $a_{x} E_{I} a_{y}$.

We now show that we can construct a scheme satisfying (1)-(6) above. Suppose $q_{k}, s_{k, i}$ and $s_{t}$ have been defined for all $k \leq n$ and $t \in 2^{\leq n}$. It is enough to define $s_{n+1, i}$ satisfying (4)-(6). Define

$$
f_{q_{n}}: 2^{\omega} \rightarrow[0, \infty]: f_{q_{n}}(x)=\sum_{k=0}^{\infty} \frac{x(k)}{q_{n}+k+1} .
$$


It is clear that $f_{q_{n}}\left(N_{0^{k}}\right)$ is dense in $[0, \infty]$ for all $k \in \omega$. Let

$$
A^{\prime}=\left\{x \in A: \lim _{k \rightarrow \infty} \mu\left(A_{(x \nmid k)}\right) \rightarrow 1\right\}
$$

i.e, the set of points in $A$ of density 1 . By the Lebesgue density theorem $[9,17.9]$ we have $\mu\left(A \backslash A^{\prime}\right)=0$. Let $A^{\prime \prime}=\bigcap_{t \in 2^{n}} A_{\left(s_{t}\right)}^{\prime}$ and note that by (6) we have $\mu\left(A^{\prime \prime}\right)>0$. Thus the set of differences $A^{\prime \prime}-A^{\prime \prime}$ contains a neighborhood of $0^{\infty}$ by $[9,17.13]$. It follows that there are $x_{0}, x_{1} \in A^{\prime \prime}$ such that

$$
\frac{1}{n+2} \leq \sum_{k=0}^{\infty} \frac{\left|x_{0}(k)-x_{1}(k)\right|}{q_{n}+k+1} \leq \frac{2}{n+2}
$$

Since all points in $A_{\left(s_{t}\right)}^{\prime}$ have density 1 in $A_{\left(s_{t}\right)}^{\prime}$ there is some $k_{0} \in \omega$ such that

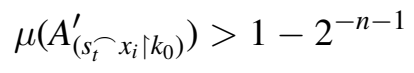

for all $t \in 2^{n}$. Defining $s_{n+1, i}=x_{i} \uparrow k_{0}$, it is then clear that (4)-(6) holds.

Proof of Proposition 1 As the proof easily relativizes, assume that $a=0$. We proceed exactly as in [3, Proposition 4.2]. Suppose $A \subseteq P\left(2^{\omega}\right)$ is a $\Sigma_{2}^{1}$ m.o. family. Recall from [10] and [3, p. 1406] that there is a Borel function $2^{\omega} \rightarrow P\left(2^{\omega}\right): x \mapsto \mu^{x}$ such that

$$
x E_{I} y \Longrightarrow \mu^{x} \approx \mu^{y}
$$

and

$$
x \#_{I} y \Longrightarrow \mu^{x} \perp \mu^{y} .
$$

Define as in [3, Proposition 4.2] a relation $Q \subseteq 2^{\omega} \times P\left(2^{\omega}\right)^{\omega}$ by

$$
Q\left(x,\left(\nu_{n}\right)\right) \Longleftrightarrow(\forall n)\left(\nu_{n} \in A \wedge \nu_{n} \not \perp \mu^{x}\right) \wedge(\forall \mu)\left(\mu \not \perp \mu^{x} \longrightarrow(\exists n) \nu_{n} \not \perp \mu\right)
$$

and note that this is $\Sigma_{2}^{1}$ when $A$ is. Note that $Q\left(x,\left(\nu_{n}\right)\right)$ precisely when $\left(\nu_{n}\right)$ enumerates the measures in $A$ not orthogonal to $\mu^{x}$ (this set is always countable, see [10, Theorem 3.1].) Since $A$ is maximal, each section $Q_{x}$ is non-empty, and so we can uniformize $Q$ with a (total) function $f: 2^{\omega} \rightarrow p\left(2^{\omega}\right)^{\omega}$ having a $\Delta_{2}^{1}$ graph. Note that assignment

$$
x \mapsto A(x)=\left\{f(x)_{n}: n \in \mathcal{N}\right\}
$$

is invariant on the $E_{I}$ classes.

If there is a Cohen real over $L$ it follows from [6] that $f$ is Baire measurable. Since $E_{I}$ is a turbulent equivalence relation (in the sense of Hjorth, see e.g. [10]) the map $x \mapsto A(x)$ must be constant on a comeagre set. But this contradicts that all $E_{I}$ classes are meagre.

If on the other hand there is a random real over $L$, then $f$ is Lebesgue measurable by [6]. Let $F \subseteq 2^{\omega}$ be a closed set with positive measure on which $f$ is continuous, and let $g: 2^{\omega} \rightarrow F$ be a Borel reduction of $E_{I}$ to $E_{I}\left\lceil F\right.$. Note that $x \mapsto A(g(x))$ is then an $E_{I}$-invariant Borel assignment of countable subsets of $p\left(2^{\omega}\right)$, and so since $E_{I}$ is turbulent the function $f \circ g$ must be constant on a comeagre set. This again contradicts that all $E_{I}$ classes are meagre. 


\section{$3 \quad \Delta_{3}^{1}$ w.o. of the reals, $\Pi_{2}^{1}$ m.o. family, no $\Sigma_{\mathbf{2}}^{\mathbf{1}}$ m.o. families with $\mathfrak{b}=\mathfrak{c}=\omega_{3}$}

We proceed with the proof of Theorem 1. We will use a modification of the model constructed in [2]. We work over the constructible universe $L$. Recall that a transitive $Z F^{-}$model is suitable if $\omega_{3}^{\mathcal{M}}$ exists and $\omega_{3}^{\mathcal{M}}=\omega_{3}^{L^{\mathcal{M}}}$. If $\mathcal{M}$ is suitable then also $\omega_{1}^{\mathcal{M}}=\omega_{1}^{L^{\mathcal{M}}}$ and $\omega_{2}^{\mathcal{M}}=\omega_{2}^{L^{\mathcal{M}}}$. Our construction can be considered a two stage process - a preliminary stage and a coding stage. In the preliminary stage (Steps 0 through 3 below), we obtain a generic extension of $L$ over which we can perform a finite support iteration of length $\omega_{3}$ (coding stage), leading to a model satisfying Theorem 1.

Fix a $\nabla_{\omega_{2}}\left(\operatorname{cof}\left(\omega_{1}\right)\right)$ sequence $\left\langle G_{\xi}: \xi \in \omega_{2} \cap \operatorname{cof}\left(\omega_{1}\right)\right\rangle$ which is $\Sigma_{1}$-definable over $L_{\omega_{2}}$. For $\alpha<\omega_{3}$, let $W_{\alpha}$ be the $L$-least subset of $\omega_{2}$ coding $\alpha$ and for $1<\alpha<\omega_{3}$ let $S_{\alpha}=\left\{\xi \in \omega_{2} \cap \operatorname{cof}\left(\omega_{1}\right): G_{\xi}=W_{\alpha} \cap \xi \neq \emptyset\right\}$. Then $\vec{S}=\left\langle S_{\alpha}: 1<\alpha<\omega_{3}\right\rangle$ is a sequence of stationary subsets of $\omega_{2} \cap \operatorname{cof}\left(\omega_{1}\right)$, which are mutually almost disjoint. Let $S_{-1}=\left\{\xi \in \omega_{2} \cap \operatorname{cof}\left(\omega_{1}\right): G_{\xi}=\emptyset\right\}$. Note that $S_{-1}$ is a stationary subset of $\omega_{2} \cap \operatorname{cof}\left(\omega_{1}\right)$ which is disjoint from all $S_{\alpha}$ 's.

Step 0 . For every $\alpha$ such that $\omega_{2} \leq \alpha<\omega_{3}$ shoot a club $C_{\alpha}$ disjoint from $S_{\alpha}$ via the poset $\mathbb{P}_{\alpha}^{0}$, consisting of all closed subsets of $\omega_{2}$ which are disjoint from $S_{\alpha}$ with the extension relation being end-extension, and let $\mathbb{P}^{0}=\prod_{\alpha<\omega_{3}} \mathbb{P}_{\alpha}^{0}$ be the direct product of the $\mathbb{P}_{\alpha}^{0}$ 's with supports of size $\omega_{1}$, where for $\alpha \in \omega_{2}, \mathbb{P}_{\alpha}^{0}$ is the trivial poset. Then $\mathbb{P}^{0}$ is countably closed, $\omega_{2}$-distributive (the proof of which uses the stationarity of $S_{-1}$ ) and $\omega_{3}$-c.c.

Step 1. We begin by fixing some notation. Let $\operatorname{Lim}^{\prime}\left(\omega_{2}\right)$ be the set of all limit ordinals $\xi$ in $\omega_{2}$ which can be presented in the form $\xi=\omega \cdot \omega \cdot \alpha^{\prime \prime}$ for some $\alpha^{\prime \prime} \geq 0$. Let $\operatorname{Lim}^{\prime}\left(\omega_{3}\right)$ be the set of all limit ordinals $\alpha$ in $\omega_{3}$ which can be presented in the form $\alpha=\omega^{2} \cdot \alpha^{\prime}+\xi$, where $\alpha^{\prime}>0$ and $\xi \in \operatorname{Lim}^{\prime}\left(\omega_{2}\right)$. Also, whenever $k \in \omega, X$ is a set of ordinals and $j \in k$, let $I_{j}^{k}(X)=\{\gamma: k \cdot \gamma+j \in X\}$. In particular, let $\operatorname{Even}(X)=I_{0}^{2}(X)=\{\gamma: 2 \cdot \gamma \in X\}$.

Let $\alpha \in\left[\omega_{2}, \omega_{3}\right)$. Then $\alpha=\alpha_{0}+\omega \cdot k+m$ for some $\alpha_{0} \in \operatorname{Lim}^{\prime}\left(\omega_{3}\right), k, m \in \omega$. Then, let $D_{\alpha}=D_{\alpha}^{k}$ be a subset of $\omega_{2}$ coding the tuple $\left(C_{\alpha}, W_{\alpha},\left\langle W_{\alpha_{0}+\omega \cdot j}\right\rangle_{j \in k+1}\right)$. More precisely, let $D_{\alpha}=D_{\alpha}^{k}$ be a subset of $\omega_{2}$ such that $I_{j}^{k+3}\left(D_{\alpha}\right)=W_{\alpha_{0}+\omega \cdot j}$ for $j \in k+1, I_{k+1}^{k+3}\left(D_{\alpha}\right)=D_{\alpha}$ and $I_{k+2}^{k+3}\left(D_{\alpha}\right)=C_{\alpha}$. Now let

$$
E_{\alpha}=E_{\alpha}^{k}=\left\{\mathcal{M} \cap \omega_{2}: \mathcal{M} \prec L_{\alpha+\omega_{2}+1}\left[D_{\alpha}\right], \omega_{1} \cup\left\{D_{\alpha}\right\} \subseteq \mathcal{M}\right\} .
$$

Then $E_{\alpha}$ is a club on $\omega_{2}$. Choose $Z_{\alpha}=Z_{\alpha}^{k} \subseteq \omega_{2}$ such that $\operatorname{Even}\left(Z_{\alpha}\right)=D_{\alpha}$ and if $\beta<\omega_{2}$ is the $\omega_{2}^{\mathcal{M}}$ for some suitable model $\mathcal{M}$ such that $Z_{\alpha} \cap \beta \in \mathcal{M}$, then $\beta \in E_{\alpha}$. Then we have:

$(*)_{\alpha, k}$ : If $\beta<\omega_{2}, \mathcal{M}$ is a suitable model such that $\omega_{1} \subset \mathcal{M}, \omega_{2}^{\mathcal{M}}=\beta$, and $Z_{\alpha} \cap \beta \in \mathcal{M}$, then $\mathcal{M} \vDash \psi_{k}\left(\omega_{2}, Z_{\alpha} \cap \beta\right)$, where $\psi_{k}\left(\omega_{2}, X\right)$ is the formula " Even $(X)$ codes a triple $\left(\bar{C}, \bar{W},\left\langle\bar{W}_{j}\right\rangle_{j \in k+1}\right)$, where $\bar{W}$ and $\bar{W}_{k}$ are the $L$-least codes of ordinals $\bar{\alpha}, \bar{\alpha}_{k}<\omega_{3}$ such that $\bar{\alpha}_{k}$ is the largest limit ordinal not exceeding $\bar{\alpha}$, for $j \in k \bar{W}_{j}$ is the $L$-least code for the largest limit ordinal $\bar{\alpha}_{j}$ strictly smaller than $\bar{\alpha}_{j+1}$, and $\bar{C}$ is a club in $\omega_{2}$ disjoint from $S_{\bar{\alpha}}$ ". 
Similarly to $\vec{S}$, define a sequence $\vec{A}=\left\langle A_{\xi}: \xi<\omega_{2}\right\rangle$ of stationary subsets of $\omega_{1}$ using the "standard" $\diamond$-sequence. Code $Z_{\alpha}$ by a subset $X_{\alpha}=X_{\alpha}^{k}$ of $\omega_{1}$ with the poset $\mathbb{P}_{\alpha}^{1}$ consisting of all pairs $\left\langle s_{0}, s_{1}\right\rangle \in$ $\left[\omega_{1}\right]^{<\omega_{1}} \times\left[Z_{\alpha}\right]^{<\omega_{1}}$ where $\left\langle t_{0}, t_{1}\right\rangle \leq\left\langle s_{0}, s_{1}\right\rangle$ iff $s_{0}$ is an initial segment of $t_{0}, s_{1} \subseteq t_{1}$ and $t_{0} \backslash s_{0} \cap A_{\xi}=\emptyset$ for all $\xi \in s_{1}$. Then $X_{\alpha}$ satisfies the following condition:

$(* *)_{\alpha, k}$ : If $\omega_{1}<\beta \leq \omega_{2}$ and $\mathcal{M}$ is a suitable model such that $\omega_{2}^{\mathcal{M}}=\beta$ and $\left\{X_{\alpha}\right\} \cup \omega_{1} \subset \mathcal{M}$, then $\mathcal{M} \vDash \phi_{k}\left(\omega_{1}, \omega_{2}, X_{\alpha}\right)$, where $\phi_{k}\left(\omega_{1}, \omega_{2}, X\right)$ is the formula: "Using the sequence $\vec{A}, X$ almost disjointly codes a subset $\bar{Z}$ of $\omega_{2}$, such that $\operatorname{Even}(\bar{Z})$ codes a triple $\left(\bar{C}, \bar{W},\left\langle\bar{W}_{j}\right\rangle_{j \in k+1}\right)$, where $\bar{W}$ and $\bar{W}_{k}$ are the $L$-least codes of ordinals $\bar{\alpha}, \bar{\alpha}_{k}<\omega_{3}$ such that $\bar{\alpha}_{k}$ is the largest limit ordinal not exceeding $\bar{\alpha}$, for $j \in k \bar{W}_{j}$ is the $L$-least code for the largest limit ordinal $\bar{\alpha}_{j}$ strictly smaller than $\bar{\alpha}_{j+1}$, and $\bar{C}$ is a club in $\omega_{2}$ disjoint from $S_{\bar{\alpha}}$ ".

Let $\mathbb{P}^{1}=\prod_{\alpha<\omega_{3}} \mathbb{P}_{\alpha}^{1}$, where $\mathbb{P}_{\alpha}^{1}$ is the trivial poset for all $\alpha \in \omega_{2}$, with countable support. Then $\mathbb{P}^{1}$ is countably closed and has the $\omega_{2}$-c.c.

Finally we force a localization of the $X_{\alpha}$ 's. Fix $\phi_{k}$ as in $(* *)_{\alpha, k}$ and define the poset $\mathcal{L}_{k}\left(X, X^{\prime}\right)$ similarly to the poset defined in [2, Definition 1] as follows.

Definition 3.1 Let $X, X^{\prime} \subset \omega_{1}$ be such that $\phi_{k}\left(\omega_{1}, \omega_{2}, X\right)$ and $\phi_{k}\left(\omega_{1}, \omega_{2}, X^{\prime}\right)$ hold in any suitable model $\mathcal{M}$ with $\omega_{1}^{\mathcal{M}}=\omega_{1}^{L}$ containing $X$ and $X^{\prime}$, respectively. Then let $\mathcal{L}_{k}\left(X, X^{\prime}\right)$ be the poset of all functions $r:|r| \rightarrow 2$, where the domain $|r|$ of $r$ is a countable limit ordinal such that:

(1) if $\gamma<|r|$ then $\gamma \in X$ iff $r(3 \gamma)=1$

(2) if $\gamma<|r|$ then $\gamma \in X^{\prime}$ iff $r(3 \gamma+1)=1$

(3) if $\gamma \leq|r|, \mathcal{M}$ is a countable suitable model containing $r \uparrow \gamma$ as an element and $\gamma=\omega_{1}^{\mathcal{M}}$, then $\mathcal{M} \vDash \phi_{k}\left(\omega_{1}, \omega_{2}, X \cap \gamma\right) \wedge \phi_{k}\left(\omega_{1}, \omega_{2}, X^{\prime} \cap \gamma\right)$.

The extension relation is end-extension.

For every $\alpha \in \operatorname{Lim}^{\prime}\left(\omega_{3}\right), k, m \in \omega$, let $\mathbb{P}_{\alpha, k, m}^{2}=\mathcal{L}_{k}\left(X_{\alpha+\omega \cdot k+m}, X_{\alpha+\omega \cdot k}\right)$ and for $\alpha \in \omega_{2}$, let $\mathbb{P}_{\alpha}^{2}$ be the trivial poset. Let

$$
\mathbb{P}^{2}=\left(\prod_{\alpha \in L i m^{\prime}\left(\omega_{3}\right)} \prod_{k, m \in \omega} \mathbb{P}_{\alpha, k, m}^{2}\right) \times\left(\prod_{\alpha \in \omega_{2}} \mathbb{P}_{\alpha}^{2}\right)
$$

with countable supports. Note that the poset $\mathbb{P}_{\alpha, k, m}^{2}$, where $\alpha \in \operatorname{Lim}^{\prime}\left(\omega_{3}\right), k, m \in \omega$, produces a generic function in ${ }^{\omega_{1}} 2$ (of $L^{\mathbb{P}^{0} * \mathbb{P}^{1}}$ ), which is the characteristic function of a subset $Y_{\alpha, k, m}$ of $\omega_{1}$ with the following property:

$(* * *)_{\alpha, k}:$ For every $\beta<\omega_{1}$ and any suitable $\mathcal{M}$ such that $\omega_{1}^{\mathcal{M}}=\beta$ and $Y_{\alpha, k, m} \cap \beta$ belongs to $\mathcal{M}$, we have $\mathcal{M} \vDash \phi_{k}\left(\omega_{1}, \omega_{2}, X_{\alpha+\omega \cdot k+m} \cap \beta\right) \wedge \phi_{k}\left(\omega_{1}, \omega_{2}, X_{\alpha+\omega \cdot k} \cap \beta\right)$. 
Similarly to the proof of $\left[2\right.$, Lemma 1] one can show that $\mathbb{P}_{0}:=\mathbb{P}^{0} * \mathbb{P}^{1} * \mathbb{P}^{2}$ is $\omega$-distributive.

Step 3. We proceed with the coding stage of our construction. We will define a finite support iteration $\left\langle\mathbb{P}_{\alpha}, \dot{\mathbb{Q}}_{\beta}: \alpha \leq \omega_{3}, \beta<\omega_{3}\right\rangle$ such that $\mathbb{P}_{0}=\mathbb{P}^{0} * \mathbb{P}^{1} * \mathbb{P}^{2}$, for every $\alpha<\omega_{3}$, $\dot{\mathbb{Q}}_{\alpha}$ is a $\mathbb{P}_{\alpha}$-name for a $\sigma$-centered poset, in $L^{\mathbb{P}_{3}}$ there is a $\Delta_{3}^{1}$-definable wellorder of the reals, a $\Pi_{2}^{1}$-definable maximal family of orthogonal measures, there are no $\boldsymbol{\Sigma}_{\mathbf{2}}^{\mathbf{1}}$-definable maximal families of orthogonal measures and $\mathfrak{b}=\mathfrak{c}=\omega_{3}$. Along the iteration for every $\alpha<\omega_{3}$, we will define in $V^{\mathbb{P}_{\alpha}}$ a set $O_{\alpha}$ of codes for measures and a set $A_{\alpha}$ of ordinals. Every $\mathbb{Q}_{\alpha}$ will add a generic real, whose $\mathbb{P}_{\alpha}$-name will be denoted $\dot{u}_{\alpha}$ and similarly to the proof of [2, Lemma 2] one can prove that $L\left[G_{\alpha}\right] \cap{ }^{\omega} \omega=L\left[\left\langle\dot{u}_{\xi}^{G_{\alpha}}: \xi<\alpha\right\rangle\right] \cap{ }^{\omega} \omega$ for every $\mathbb{P}_{\alpha}$-generic filter $G_{\alpha}$. This gives a canonical wellorder of the reals in $L\left[G_{\alpha}\right]$ which depends only on the sequence $\left\langle\dot{u}_{\xi}: \xi<\alpha\right\rangle$, whose $\mathbb{P}_{\alpha}$-name will be denoted by $\dot{<}_{\alpha}$. We can additionally arrange that for $\alpha<\beta,<_{\alpha}$ is an initial segment of $<_{\beta}$, where $<_{\alpha}=\dot{<}_{\alpha}^{G_{\alpha}}$ and $<_{\beta}=\dot{<}_{\beta}^{G_{\beta}}$. Then if $G$ is a $\mathbb{P}_{\omega_{3}}$-generic filter over $L$, then $<^{G}=\bigcup\left\{\dot{<}_{\alpha}^{G}: \alpha<\omega_{3}\right\}$ will be the desired wellorder of the reals.

We will need some more notation. If $x, y$ are reals in $L\left[G_{\alpha}\right]$ such that $x<_{\alpha} y$, let $x * y=\{2 n: n \in$ $x\} \cup\{2 n+1: n \in y\}$ and $\Delta(x * y)=\{2 n+2: n \in x * y\} \cup\{2 n+1: n \notin x * y\}$. For every $\alpha \in\left[\omega_{2}, \omega_{3}\right)$, let $\dot{F}_{\alpha}^{0}, \dot{F}_{\alpha}^{1}$ be $\mathbb{P}_{\alpha}$-names for nicely definable bijections $F_{\alpha}^{0}: 2^{\omega} \rightarrow p_{c}\left(2^{\omega}\right)$ and $F_{\alpha}^{1}:\left(2^{\omega}\right)^{\omega} \rightarrow 2^{\omega}$ in $L\left[G_{\alpha}\right]$, respectively, such that whenever $\alpha<\beta, i \in\{0,1\}$ we have $F_{\alpha}^{i} \subseteq F_{\beta}^{i}$. For example, identifying $p_{c}\left(2^{\omega}\right)$ with countable sequences of reals, let $\left(F_{\alpha}^{0}\right)^{-1}, F_{\alpha}^{1}$ be simply Cantor diagonalization. For every $\nu \in\left[\omega_{2}, \omega_{3}\right)$ let $i_{\nu}: \nu \cup\{\langle\xi, \eta\rangle: \xi<\eta<\nu\} \rightarrow \operatorname{Lim}^{\prime}\left(\omega_{2}\right)$ be a fixed bijection and let $\vec{B}=\left\langle B_{\zeta, m}: \zeta<\omega_{1}, m \in \omega\right\rangle$ be a nicely definable sequence of almost disjoint subsets of $\omega$.

Suppose $\mathbb{P}_{\alpha}$ has been defined and fix a $\mathbb{P}_{\alpha}$-generic filter $G_{\alpha}$.

Case A. Suppose $\alpha \in \operatorname{Lim}^{\prime}\left(\omega_{3}\right)$, i.e. $\alpha=\omega^{2} \cdot \alpha^{\prime}+\xi$ for some $\alpha^{\prime}>0, \xi \in \operatorname{Lim}^{\prime}\left(\omega_{2}\right)$. Let $\nu=$ o.t. $\left(\dot{<}_{\omega_{2} \cdot \alpha^{\prime}}^{G_{\alpha}}\right)$, $i=i_{\nu}$.

Case A.1. If $i^{-1}(\xi)=\left\langle\xi_{0}, \xi_{1}\right\rangle$ for some $\xi_{0}<\xi_{1}<\nu$, let $x_{\xi_{0}}$ and $x_{\xi_{1}}$ be the $\xi_{0}$-th and $\xi_{1}$-th reals in $L\left[G_{\omega_{2} \cdot \alpha^{\prime}}\right]$ according to the wellorder $\dot{<}_{\omega_{2} \cdot \alpha^{\prime}}^{G_{\alpha}}$. In $L^{\mathbb{P}_{\alpha}}$ let

$$
\mathbb{Q}_{\alpha}=\left\{\left\langle s_{0}, s_{1}\right\rangle: s_{0} \in[\omega]^{<\omega}, s_{1} \in\left[\bigcup_{m \in \Delta\left(x_{\xi_{0}} * x_{\xi_{1}}\right)} Y_{\alpha+m} \times\{m\}\right]^{<\omega}\right\},
$$

where $\left\langle t_{0}, t_{1}\right\rangle \leq\left\langle s_{0}, s_{1}\right\rangle$ if and only if $s_{1} \subseteq t_{1}, s_{0}$ is an initial segment of $t_{0}$ and $\left(t_{0} \backslash s_{0}\right) \cap B_{\zeta, m}=\emptyset$ for all $\langle\zeta, m\rangle \in s_{1}$. Let $u_{\alpha}$ be the generic real added by $\mathbb{Q}_{\alpha}, A_{\alpha}=\alpha+\omega \backslash \Delta\left(x_{\xi_{0}} * x_{\xi_{1}}\right)$ and $O_{\alpha}=\emptyset$. For every $n \geq 1$, let $\mathbb{Q}_{\alpha+n}$ be the poset (in $L^{\mathbb{P}_{\alpha+n}}$ ) adding a dominating real $u_{\alpha+n}$, which is defined in Case B below and let $A_{\alpha+n}=O_{\alpha+n}=\emptyset$.

Case A.2. Suppose $i^{-1}(\xi)=\zeta \in \nu$. Consider the $\zeta$-th real $x_{\zeta}$ according to the wellorder $\dot{<}_{\omega_{2} \cdot \alpha^{\prime}}^{G_{\alpha}}$. Let $F_{\omega_{2} \cdot \alpha^{\prime}}^{0}=\dot{F}_{\omega_{2} \cdot \alpha^{\prime}}^{0}\left[G_{\alpha}\right]$ and let $f_{\alpha}=\left(F_{\omega_{2} \cdot \alpha^{\prime}}^{0}\right)\left(x_{\zeta}\right)$. 
Case A.2.1. If $f_{\alpha}$ is not a code for a measure orthogonal to $O_{\alpha}^{\prime}=\bigcup_{\gamma<\alpha} O_{\gamma}$, for every $n \in \omega$ recursively define in $L^{\mathbb{P}_{\alpha+n}}, \mathbb{Q}_{\alpha+n}$ to be the poset for adding a dominating real defined in Case B below and let $A_{\alpha+n}=O_{\alpha+n}=\emptyset$.

Case A.2.2. Otherwise, i.e. in case $f_{\alpha}$ is a code for a measure orthogonal to $O_{\alpha}^{\prime}=\bigcup_{\gamma<\alpha} O_{\gamma}$, define the poset $\mathbb{Q}_{\alpha+n}$, the set of codes for measures $O_{\alpha+n}$ and the set of ordinals $A_{\alpha+n}$ in $L^{\mathbb{P}_{\alpha+n}}$ recursively as follows.

- $\mathbb{Q}_{\alpha}$ almost disjointly, via the sequence $\vec{B}$, codes the sequence $\left\langle Y_{\alpha+m}: m \in \Delta\left(x_{\zeta}\right)\right\rangle$. More precisely let

$$
\mathbb{Q}_{\alpha}=\left\{\left\langle s_{0}, s_{1}\right\rangle: s_{0} \in[\omega]^{<\omega}, s_{1} \in\left[\bigcup_{m \in \Delta\left(x_{\zeta}\right)} Y_{\alpha+m} \times\{m\}\right]^{<\omega}\right\},
$$

where $\left\langle t_{0}, t_{1}\right\rangle \leq\left\langle s_{0}, s_{1}\right\rangle$ if and only if $s_{1} \subseteq t_{1}, s_{0}$ is an initial segment of $t_{0}$ and $\left(t_{0} \backslash s_{0}\right) \cap B_{\zeta, m}=\emptyset$ for all $\langle\zeta, m\rangle \in s_{1}$. Let $u_{\alpha}$ be the generic real added by $\mathbb{Q}_{\alpha}, A_{\alpha}=\alpha+\omega \backslash \Delta\left(u_{\alpha}\right)$.

- Let $n \geq 1$. Suppose $\mathbb{Q}_{\alpha+(n-1)}$ has been defined and adds a real $u_{\alpha+(n-1)}$. Then $\mathbb{Q}_{\alpha+n}$ almost disjointly, via the sequence $\vec{B}$, codes the sequence $\left\langle Y_{\alpha+\omega \cdot n+m}: m \in \Delta\left(u_{\alpha+(n-1)}\right)\right\rangle$. More precisely let

$$
\mathbb{Q}_{\alpha+n}=\left\{\left\langle s_{0}, s_{1}\right\rangle: s_{0} \in[\omega]^{<\omega}, s_{1} \in\left[\bigcup_{m \in \Delta\left(u_{\alpha+(n-1)}\right)} Y_{\alpha+\omega \cdot n+m} \times\{m\}\right]^{<\omega}\right\},
$$

where $\left\langle t_{0}, t_{1}\right\rangle \leq\left\langle s_{0}, s_{1}\right\rangle$ if and only if $s_{1} \subseteq t_{1}, s_{0}$ is an initial segment of $t_{0}$ and $\left(t_{0} \backslash s_{0}\right) \cap B_{\zeta, m}=\emptyset$ for all $\langle\zeta, m\rangle \in s_{1}$. Let $u_{\alpha+n}$ be the generic real added by $\mathbb{Q}_{\alpha+n}, A_{\alpha+n}=\alpha+\omega \cdot n+\omega \backslash \Delta\left(u_{\alpha+(n-1)}\right)$.

In $L^{\mathbb{P}_{\alpha+\omega}}$ let $\vec{u}_{\alpha}=\left(u_{n}^{\alpha}\right)_{n \in \omega}$, where $u_{0}^{\alpha}=x_{\zeta}$ and $u_{n}^{\alpha}=u_{\alpha+n-1}$ whenever $n \geq 1$. Let

$$
g_{\alpha}=\bar{r}\left(F_{\alpha+\omega}^{0}\left(u_{0}^{\alpha}\right), F_{\alpha+\omega}^{1}\left(\left(u_{n}^{\alpha}\right)_{n \geq 1}\right)\right)
$$

(see Lemma 1) and for every $n \in \omega$ let $O_{\alpha+n}=\left\{g_{\alpha}\right\}$.

Case B. Suppose either $\alpha \in \omega_{2}$, or $\alpha \in \operatorname{Lim}\left(\omega_{3}\right) \backslash \operatorname{Lim}^{\prime}\left(\omega_{3}\right)$, or $\alpha$ is a successor ordinal in $\left(\omega_{2}, \omega_{3}\right)$ which is not of the form $\alpha^{\prime}+n$ for some $\alpha^{\prime} \in \operatorname{Lim}^{\prime}\left(\omega_{3}\right), n \in \omega$. Then let $\mathbb{Q}_{\alpha}$ be the following poset for adding a dominating real:

$$
\mathbb{Q}_{\alpha}=\left\{\left\langle s_{0}, s_{1}\right\rangle: s_{0} \in \omega^{<\omega}, s_{1} \in\left[\text { o.t. }\left(\dot{<}_{\alpha}^{G_{\alpha}}\right)\right]^{<\omega}\right\},
$$

where $\left\langle t_{0}, t_{1}\right\rangle \leq\left\langle s_{0}, s_{1}\right\rangle$ if and only if $s_{0}$ is an initial segment of $t_{0}, s_{1} \subseteq t_{1}$, and $t_{0}(n)>x_{\xi}(n)$ for all $n \in \operatorname{dom}\left(t_{0}\right) \backslash \operatorname{dom}\left(s_{0}\right)$ and $\xi \in s_{1}$, where $x_{\xi}$ is the $\xi$-th real in $L\left[G_{\alpha}\right] \cap \omega^{\omega}$ according to the wellorder $\dot{<}_{\alpha}^{G_{\alpha}}$. Let $A_{\alpha}=\emptyset, O_{\alpha}=\emptyset$.

With this the definition of $\mathbb{P}_{\omega_{3}}$ is complete. Similarly to [2, Lemma 3] one can show that if $G$ is $\mathbb{P}_{\omega_{3}}$-generic and $\xi \in \bigcup_{\alpha \in \omega_{3}} \dot{A}_{\alpha}^{G}$, then in $L[G]$ there is no real coding a stationary kill of $S_{\xi}$. We will refer to this fact, as

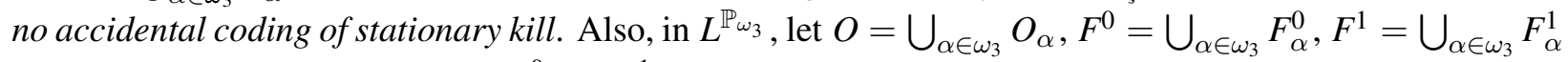
and for $\vec{z}=\left(z_{n}\right)_{n \in \omega}$, let $\mathcal{R}(\vec{z})=\bar{r}\left(F^{0}\left(z_{0}\right), F^{1}\left(\left(z_{n}\right)_{n \geq 1}\right)\right)$ (see Lemma 1$)$. 
Lemma 3.2 Let $G$ be $\mathbb{P}_{\omega_{3}}$ generic, $g=\mathcal{R}(\vec{z})$ for some $\vec{z}=\left(z_{n}\right)_{n \in \omega}$. Then $g \in O$ if and only if for every countable suitable model $\mathcal{M}$ such that $g \in \mathcal{M}$ there is $\bar{\alpha}<\omega_{3}^{\mathcal{M}}$ such that for all $n \in \omega, S_{\bar{\alpha}+\omega \cdot n+m}$ is non-stationary in $\left(L\left[z_{n+1}\right]\right)^{\mathcal{M}}$ for all $m \in \Delta\left(z_{n}\right)$.

Proof Suppose $g=\mathcal{R}(\vec{z})$ and for every countable suitable model $\mathcal{M}$ such that $g \in \mathcal{M}$, there is $\bar{\alpha}<\omega_{3}^{\mathcal{M}}$ with the above property. By the Löweinheim-Skolem theorem, the same holds in $\mathbb{H}_{\Theta}^{\mathbb{P}}$, where $\Theta$ is sufficiently large and $\mathbb{H}_{\Theta}^{\mathbb{P}}$ denotes the set of all sets hereditary of cardinality $<\Theta$. Thus there is $\alpha<\omega_{3}$ such that for all $n \in \omega, m \in \Delta\left(z_{n}\right), L_{\Theta}\left[z_{n+1}\right] \vDash\left(S_{\alpha+\omega \cdot n+m}\right.$ is not stationary $)$. Then in particular for some $n>0, m \in \omega$ the stationary kill of $S_{\alpha+\omega \cdot n+m}$ is coded by a real. Since there is no accidental coding of stationary kill, $\mathbb{Q}_{\alpha+n}$ adds a real $u_{\alpha+n}\left(=u_{n+1}^{\alpha}\right)$ coding a stationary kill of $S_{\alpha+\omega \cdot n+m}$ for all $m \in \Delta\left(u_{n}^{\alpha}\right)$, while there are no reals coding the stationary kill of $S_{\alpha+\omega \cdot n+m}$ for $m \notin \Delta\left(u_{n}^{\alpha}\right)$. Therefore $\Delta\left(u_{n}^{\alpha}\right)=\Delta\left(z_{n}\right)$ for all $n$, and so $\vec{u}_{\alpha}=\vec{z}$, which implies $g=g_{\alpha} \in O$.

On the other hand, suppose $g \in \mathcal{R}(\vec{z}) \in O$. Thus $g=g_{\alpha}=\mathcal{R}\left(\vec{u}_{\alpha}\right)$ and since $\mathcal{R}$ is injective $\vec{u}_{\alpha}=\vec{z}$. Suppose $\mathcal{M}$ is a suitable model which contains $g$. Then by definition of $\bar{r}$ we have that $F^{0}\left(u_{0}^{\alpha}\right)$ and $F^{1}\left(\left(u_{n}^{\alpha}\right)_{n \geq 1}\right)$ are also in $\mathcal{M}$. Since $F^{0}, F^{1}$ are nicely definable, $\mathcal{M}$ contains also $\vec{u}_{\alpha}$. Therefore for all $n \in \omega, m \in \Delta\left(u_{n}^{\alpha}\right)$ also the sets $Y_{\alpha+\omega \cdot n+m} \cap \omega_{1}^{\mathcal{M}}$ are in $\mathcal{M}$. Thus in particular, $\mathcal{M}$ contains the sets $X_{\alpha+\omega \cdot n+m} \cap \omega_{1}^{\mathcal{M}}$ for all $n \in \omega, m \in \Delta\left(u_{n}^{\alpha}\right)$. Fix $n, m \in \Delta\left(u_{n}^{\alpha}\right)$. By definition of $\mathcal{L}_{n}\left(X_{\alpha+\omega \cdot n+m}, X_{\alpha+\omega \cdot n}\right)$ we have that for every $m \in \Delta\left(u_{n}^{\alpha}\right)$, in $\mathcal{M}$, using the sequence $\vec{A}$, the set $X_{\alpha+\omega \cdot n+m} \cap \omega_{1}$ almost disjointly codes a subset $\bar{Z}^{n, m}$ of $\omega_{2}$, whose even part codes a triple $\left.\left(C^{n, m}, W^{n, m},\left\langle W_{j}^{n, m}\right\rangle_{j \in n+1}\right\rangle\right)$, where $W^{n, m}, W_{n}^{n, m}$ are the $L$-least codes of ordinals $\alpha^{n, m}, \alpha_{n}^{n, m}$ in $\omega_{3}$ such that $\alpha_{n}^{n, m}$ is the largest limit ordinal not exceeding $\alpha^{n, m}$ and for every $j \in n$, $\alpha_{j}^{n, m}$ is the largest limit ordinal strictly smaller than $\alpha_{j+1}^{m, n}$. It remains to observe that for every $n_{1}<n_{2}$, $m_{1}, m_{2}$ in $\omega$, we have $W_{j}^{n_{1}, m_{1}}=W_{j}^{n_{2}, m_{2}}$ whenever $j \leq n_{1}$. Therefore $\alpha_{0}^{n, m}$ does not depend on $n, m$ and so $\bar{\alpha}=\alpha_{0}^{n, m}$ is the desired ordinal.

Therefore $O$ has indeed a $\Pi_{2}^{1}$ definition. We will show that $O$ is maximal in $p_{c}\left(2^{\omega}\right)$. Indeed, suppose in $L^{\mathbb{P} \omega_{3}}$ there is a code $f$ for a measure orthogonal to every measure in the family $\bar{O}=\left\{\mu_{g}: g \in O\right\}$. Choose $\alpha$ minimal such that $\alpha=\omega_{2} \cdot \alpha^{\prime}+\xi$ for some $\alpha^{\prime}>0, \xi \in \operatorname{Lim}^{\prime}\left(\omega_{2}\right)$ and such that $f \in L\left[G_{\omega_{2} \cdot \alpha^{\prime}}\right]$. Let $\nu=$ o.t. $\left(\dot{<}_{\omega_{2} \cdot \alpha^{\prime}}^{G_{\alpha}}\right)$ and let $i=i_{\nu}$. Then $x=\left(F_{\omega_{2} \cdot \alpha^{\prime}}^{0}\right)^{-1}(f)$ is the $\zeta$-th real according to the wellorder $\dot{<}_{\omega_{2} \cdot \alpha^{\prime}}^{G_{\alpha}}$ for some $\zeta \in \nu$, which implies that for some $\xi \in \operatorname{Lim}^{\prime}\left(\omega_{2}\right), i^{-1}(\xi)=\zeta$. But then $x_{\zeta}=x$ is the code of a measure orthogonal to $O_{\alpha}^{\prime}$ and so by construction $O_{\alpha}$ contains the code of a measure equivalent to $\mu_{f}$, which is a contradiction. To obtain a $\Pi_{2}^{1}$-definable m.o. family in $L^{\mathbb{P} \omega_{3}}$ consider the union of $\bar{O}=\left\{\mu_{g}: g \in O\right\}$ with the set of all point measures. Just as in [2] one can show that $<$ is indeed a $\Delta_{3}^{1}$-definable wellorder of the reals.

Since $\mathbb{P}_{\omega_{3}}$ is a finite support iteration, we have added Cohen reals along the iteration cofinally often. Thus for every real $a$ in $L^{\mathbb{P}_{\omega_{3}}}$ there is a Cohen real over $L[a]$ and so by Proposition 1 in $L^{\mathbb{P}_{\omega_{3}}}$ there are no $\Sigma_{2}^{1}$ m.o. families. Also note that since cofinally often we have added dominating reals, $L^{\mathbb{P} \omega_{3}} \vDash \mathfrak{b}=\omega_{3}$. 


\section{$4 \quad \Delta_{3}^{1}$ w.o. of the reals, a $\Pi_{2}^{1}$ m.o. family, no $\Sigma_{2}^{1}$ m.o. families with $\mathfrak{d}=\omega_{1}$ and $\mathfrak{c}=\omega_{2}$}

In this section we establish the proof of Theorem 2. The model is obtained as a modification of the iteration construction developed in [1]. We restate the definitions of the posets used in this construction. For a more detailed account of their properties see [1]. We work over the constructible universe $L$. For the remainder of this section, we will say that a transitive $Z F^{-}$model is suitable, if $\omega_{2}^{\mathcal{M}}$ exists and $\omega_{2}^{\mathcal{M}}=\omega_{2}^{\mathcal{M}^{L}}$.

If $S \subseteq \omega_{1}$ is a stationary, co-stationary set, then by $Q(S)$ denote the poset of all countable closed subsets of $\omega_{1} \backslash S$ with the extension relation being end-extension. Recall that $Q(S)$ is $\omega_{1} \backslash S$-proper, $\omega$-distributive and adds a club disjoint from $S$ (see [1], [5]). For the proof of Theorem 2 we use the form of localization defined in [1, Definition 1]. That is, if $X \subseteq \omega_{1}$ and $\phi\left(\omega_{1}, X\right)$ is a $\Sigma_{1}$-sentence with parameters $\omega_{1}, X$ which is true in all suitable models containing $\omega_{1}$ and $X$ as elements, then let $\mathcal{L}(\phi)$ be the poset of all functions $r:|r| \rightarrow 2$, where the domain $|r|$ of $r$ is a countable limit ordinal, such that

(1) if $\gamma<|r|$ then $\gamma \in X$ iff $r(2 \gamma)=1$

(2) if $\gamma \leq|r|, \mathcal{M}$ is a countable, suitable model containing $r \uparrow \gamma$ as an element and $\gamma=\omega_{1}^{\mathcal{M}}$, then $\phi(\gamma, X \cap \gamma)$ holds in $\mathcal{M}$.

The extension relation is end-extension. Recall that $\mathcal{L}(\phi)$ has a countably closed dense subset (see [1, Remark 2]) and that if $G$ is $\mathcal{L}(\phi)$-generic and $\mathcal{M}$ is a countable suitable model containing $(\bigcup G)\lceil\gamma$ as an element, where $\gamma=\omega_{1}^{\mathcal{M}}$, then $\mathcal{M} \vDash \phi(\gamma, X \cap \gamma)$ (see [1, Lemma 2]).

We will use also the coding with perfect trees defined in [1, Definition 2]. Let $Y \subseteq \omega_{1}$ be generic over $L$ such that in $L[Y]$ cofinalities have not been changed and let $\bar{\mu}=\left\{\mu_{i}\right\}_{i \in \omega_{1}}$ be a sequence of $L$-countable ordinals such that $\mu_{i}$ is the least $\mu>\sup _{j<i} \mu_{j}, L_{\mu}[Y \cap i] \vDash Z F^{-}$and $L_{\mu} \vDash \omega$ is the largest cardinal. Say that a real $R$ codes $Y$ below $i$ if for all $j<i, j \in Y$ if and only if $L_{\mu_{j}}[Y \cap j, R] \vDash Z F^{-}$. For $T \subseteq 2^{<\omega}$ a perfect tree, let $|T|$ be the least $i$ such that $T \in L_{\mu_{i}}[Y \cap i]$. Then $\mathcal{C}(Y)$ is the poset of all perfect trees $T$ such that $R$ codes $Y$ below $|T|$, whenever $R$ is a branch through $T$, where for $T_{0}, T_{1}$ conditions in $\mathcal{C}(Y), T_{0} \leq T_{1}$ if and only if $T_{0}$ is a subtree of $T_{1}$. Recall also that $\mathcal{C}(Y)$ is proper and ${ }^{\omega} \omega$-bounding (see [1, Lemmas 7,8]).

Fix a bookkeeping function $F: \operatorname{Lim}^{\prime}\left(\omega_{2}\right) \rightarrow L_{\omega_{2}}$ and a sequence $\vec{S}=\left(S_{\beta}: \beta<\omega_{2}\right)$ of almost disjoint stationary subsets of $\omega_{1}$, defined as in [1, Lemma 14]. Thus $F$ and $\vec{S}$ are $\Sigma_{1}$-definable over $L_{\omega_{2}}$ with parameter $\omega_{1}, F^{-1}(a)$ is unbounded in $\operatorname{Lim}^{\prime}\left(\omega_{2}\right)$ for every $a \in L_{\omega_{2}}$ and whenever $\mathcal{M}, \mathcal{N}$ are suitable models such that $\omega_{1}^{\mathcal{M}}=\omega_{1}^{\mathcal{N}}$ then $F^{\mathcal{M}}, \vec{S}^{\mathcal{M}}$ agree with $F^{\mathcal{N}}, \vec{S}^{\mathcal{N}}$ on $\omega_{2}^{\mathcal{M}} \cap \omega_{2}^{\mathcal{N}}$. Also if $\mathcal{M}$ is suitable and $\omega_{1}^{\mathcal{M}}=\omega_{1}$ then $F^{\mathcal{M}}, \bar{S}^{\mathcal{M}}$ equal the restrictions of $F, \vec{S}$ to the $\omega_{2}$ of $\mathcal{M}$. Fix also a stationary subset $S$ of $\omega_{1}$ which is almost disjoint from every element of $\vec{S}$.

Recursively we will define a countable support iteration $\left\langle\mathbb{P}_{\alpha}, \dot{\mathbb{Q}}_{\beta}: \alpha \leq \omega_{2}, \beta<\omega_{2}\right\rangle$ such that in $L^{\mathbb{P}_{\omega_{2}}}$ there is a $\Delta_{3}^{1}$-definable wellorder of the reals, there is a $\Pi_{2}^{1}$ definable m.o. family, there are no $\Sigma_{\mathbf{2}}^{\mathbf{1}}$ definable m.o. families and $\mathfrak{d}=\aleph_{1}, \mathfrak{c}=\aleph_{2}$. Along the iteration for every $\alpha<\omega_{2}$, we will define in $L^{\mathbb{P}_{\alpha}}$ a set $O_{\alpha}$ of 
codes for measures and a set $A_{\alpha}$ of ordinals. Define the wellorder $<_{\alpha}$ in $L\left[G_{\alpha}\right]$ where $G_{\alpha}$ is $\mathbb{P}_{\alpha}$-generic just as in [1]. We can assume that all names for reals are nice and that for $\alpha<\beta<\omega_{2}$, all $\mathbb{P}_{\alpha}$-names for reals precede in the canonical wellorder $<_{L}$ of $L$ all $\mathbb{P}_{\beta}$-names for reals, which are not $\mathbb{P}_{\alpha}$-names. For each $\alpha<\omega_{2}$, define a wellorder $<_{\alpha}$ on the reals of $L\left[G_{\alpha}\right]$, where $G_{\alpha}$ is a $\mathbb{P}_{\alpha}$-generic as follows. If $x$ is a real in $L\left[G_{\alpha}\right]$ let $\sigma_{x}^{\alpha}$ be the $<_{L}$-least $\mathbb{P}_{\gamma}$-name for $x$, where $\gamma \leq \alpha$ is least so that $x$ has a $\mathbb{P}_{\gamma}$-name. For $x, y$ reals in $L\left[G_{\alpha}\right]$ define $x<_{\alpha} y$ if and only if $\sigma_{x}^{\alpha}<_{L} \sigma_{y}^{\alpha}$. Note that whenever $\alpha<\beta$, then $<_{\alpha}$ is an initial segment of $<_{\beta}$. Then $<^{G}=\bigcup_{\alpha<\omega_{2}} \dot{<}_{\alpha}^{G}$ will be the desired wellorder of the reals in $L[G]$, where $G$ is a $\mathbb{P}_{\omega_{2}}$-generic filter. For every $\alpha \in \omega_{2}$, let $W_{\alpha}$ be the $L$-least subset of $\omega_{1}$ coding $\alpha$. Also, for each $\alpha \in \omega_{2}$, fix $\mathbb{P}_{\alpha}$-names $\dot{F}_{\alpha}^{0}, \dot{F}_{\alpha}^{1}$ for nicely definable bijections $F_{\alpha}^{0}: 2^{\omega} \rightarrow p_{c}\left(2^{\omega}\right), F_{\alpha}^{1}:\left(2^{\omega}\right)^{\omega} \rightarrow 2^{\omega}$ in $L^{\mathbb{P}_{\alpha}}$ such that for all $i \in\{0,1\}$ and $\alpha<\beta<\omega_{2}$ in $L^{\mathbb{P}_{\beta}}$ we have $F_{\alpha}^{i} \subseteq F_{\beta}^{i}$ (e.g. take $\left(F_{\alpha}^{0}\right)^{-1}, F_{\alpha}^{1}$ to be Cantor diagonalization).

We proceed with the definition of the poset. Let $\mathbb{P}_{0}$ be the trivial poset. Suppose $\mathbb{P}_{\alpha},\left\langle O_{\gamma}: \gamma\langle\alpha\rangle\right.$ and $\left\langle A_{\gamma}: \gamma<\alpha\right\rangle$ have been defined. Let $G_{\alpha}$ be a $\mathbb{P}_{\alpha}$-generic filter.

Case A. Suppose $\alpha \in \operatorname{Lim}^{\prime}\left(\omega_{2}\right)=\left\{\alpha \in \operatorname{Lim}\left(\omega_{2}\right): \alpha=\omega \cdot \omega \cdot \alpha^{\prime \prime}\right.$ for some $\left.\alpha^{\prime \prime} \geq 0\right\}$. We will define $\mathbb{P}_{\alpha+\gamma}$ for $\gamma \in \omega \cdot \omega$ recursively as follows.

Case A.1. Suppose $F(\alpha)=\left\{\sigma_{x}^{\alpha}, \sigma_{y}^{\alpha}\right\}$ is a pair of nice names for reals in $L\left[G_{\alpha}\right]$. Let $x=\sigma_{x}^{\alpha}\left[G_{\alpha}\right]$, $y=\sigma_{y}^{\alpha}\left[G_{\alpha}\right]$.

- For every $m \in \omega$, define $\mathbb{Q}_{\alpha+m}$ in $L^{\mathbb{P}_{\alpha+m}}$ as follows. If $m \in \Delta(x * y)$ let $\mathbb{Q}_{\alpha+m}=Q\left(S_{\alpha+m}\right)$ and if $m \notin \Delta(x * y)$ let $\mathbb{Q}_{\alpha+m}$ be the random real forcing.

- In $L^{\mathbb{P}_{\alpha+\omega}}$ let $X_{\alpha+\omega}$ be a subset of $\omega_{1}$, coding $W_{\alpha}$, coding the pair $(x, y)$, coding a level of $L$ in which $\alpha$ has size at most $\omega_{1}$ and coding the generic $G_{\alpha+\omega}$, which can be regarded as a subset of an element of $L_{\omega_{2}}$. Let $\mathbb{K}_{\alpha+\omega}^{1}=\mathcal{L}\left(\phi_{\alpha+\omega}\right)$, where $\phi_{\alpha+\omega}=\phi_{\alpha+\omega}\left(\omega_{1}, X_{\alpha+\omega}\right)$ is the $\Sigma_{1}$-sentence which holds if and only if $X_{\alpha+\omega}$ codes a subset $W$ of $\omega_{1}$ and a pair $(x, y)$ of reals, such that $W$ is the $L$-least code for an ordinal $\bar{\alpha}<\omega_{2}$ and $S_{\bar{\alpha}+m}$ is non-stationary for $m \in \Delta(x * y)$. Let $\dot{X}_{\alpha+\omega}$ be a $\mathbb{P}_{\alpha+\omega}$-name for $X_{\alpha+\omega}$ and let $\dot{K}_{\alpha+\omega}^{1}$ be a $\mathbb{P}_{\alpha+\omega}$-name for $\mathbb{K}_{\alpha+\omega}^{1}$.

- Let $Y_{\alpha+\omega}$ be $\mathbb{K}_{\alpha+\omega}^{1}$-generic over $L\left[G_{\alpha+\omega}\right]$. The even part of $Y_{\alpha+\omega}$ codes $X_{\alpha+\omega}$ and so codes the generic $G_{\alpha+\omega}$. Then in $L\left[Y_{\alpha+\omega}\right]=L\left[G_{\alpha+\omega} * Y_{\alpha+\omega}\right]$, let $\mathbb{K}_{\alpha+\omega}^{2}=\mathcal{C}\left(Y_{\alpha+\omega}\right)$. Let $R_{\alpha+\omega}$ be the real added by $\mathbb{K}_{\alpha+\omega}^{2}$, let $\dot{\mathbb{K}}_{\alpha+\omega}^{2}$ be a $\mathbb{P}_{\alpha+\omega} * \dot{\mathbb{K}}_{\alpha+\omega}^{1}$-name for $\mathbb{K}_{\alpha+\omega}^{2}$ and let $\mathbb{Q}_{\alpha+\omega}=\mathbb{K}_{\alpha+\omega}^{1} * \dot{\mathbb{K}}_{\alpha+\omega}^{2}$.

- For every $\gamma \in[\alpha+\omega+1, \alpha+\omega \cdot \omega)$ let $\mathbb{Q}_{\alpha+\gamma}$ be a $\mathbb{P}_{\alpha+\gamma}$-name for the random real forcing.

Case A.2. Suppose $F(\alpha)=\left\{\sigma_{x}^{\alpha}\right\}$ for some nice name for a real $\sigma_{x}^{\alpha}$. Let $x=\sigma_{x}^{\alpha}\left[G_{\alpha}\right], f=F_{\alpha}^{0}(x)$.

Case A.2.1. If $f$ is not a code of a measure orthogonal to $O_{\alpha}^{\prime}=\bigcup_{\gamma<\alpha} O_{\gamma}$, let $\mathbb{Q}_{\alpha+\gamma}$ be a $\mathbb{P}_{\alpha+\gamma}$-name for the random real forcing, for all $\gamma \in \omega \cdot \omega$. 
Case A.2.2. If $f$ is a code of a measure orthogonal to $O_{\alpha}^{\prime}=\bigcup_{\gamma<\alpha} O_{\gamma}$, define $\mathbb{Q}_{\alpha+\gamma}$ for $\gamma \in \omega \cdot \omega$ recursively as follows. Let $\mathbb{Q}_{\alpha}$ be the trivial poset (in $L^{\mathbb{P}_{\alpha}}$ ), and let $R_{\alpha}=x$. Suppose the poset $\mathbb{P}_{\alpha+\omega \cdot n+1}$ and the real $R_{\alpha+\omega \cdot n}$ have been defined.

- For $m \geq 1$ define $\mathbb{Q}_{\alpha+\omega \cdot n+m}$ in $L^{\mathbb{P}_{\alpha+\omega \cdot n+m}}$ recursively as follows. If $m-1 \in \Delta\left(R_{\alpha+\omega \cdot n}\right)$ let $\mathbb{Q}_{\alpha+\omega \cdot n+m}=Q\left(S_{\alpha+\omega \cdot n+(m-1)}\right)$ and if $m-1 \in \Delta\left(R_{\alpha+\omega \cdot n}\right)$ let $\mathbb{Q}_{\alpha+\omega \cdot n+m}$ be the random real forcing.

- Let $G_{\alpha+\omega \cdot n+\omega}$ be a $\mathbb{P}_{\alpha+\omega \cdot n+\omega}$-generic filter. In $L\left[G_{\alpha+\omega \cdot n+\omega}\right]$ let $X_{\alpha+\omega \cdot n+\omega}$ be a subset of $\omega_{1}$ coding $W_{\alpha+\omega \cdot j}$ for $j \leq n+1$, coding the real $R_{\alpha+\omega \cdot n}$, coding a level of $L$ in which $\alpha+\omega \cdot n+\omega$ has size at most $\omega_{1}$ and coding the generic $G_{\alpha+\omega \cdot n+\omega}$. Let $\mathbb{K}_{\alpha+\omega \cdot(n+1)}^{1}$ be the poset $\mathcal{L}\left(\phi_{\alpha}^{n+1}\right)$, where $\phi_{\alpha}^{n+1}=\phi_{\alpha}^{n+1}\left(\omega_{1}, X_{\alpha+\omega \cdot(n+1)}\right)$ is the $\Sigma_{1}$-sentence which holds if and only if $X_{\alpha+\omega \cdot(n+1)}$ codes the tuple $\left\langle\bar{W}_{j}\right\rangle_{j \leq n+1}$ of subsets of $\omega_{1}$ and a real $x$, such that $\bar{W}_{n+1}$ is the $L$-least code for an ordinal $\bar{\alpha}=\bar{\alpha}_{n+1}$ and for every $j \leq n, \bar{W}_{j}$ is the $L$-least code for the largest limit $\bar{\alpha}_{j}$ strictly smaller than $\bar{\alpha}_{j+1}$, and for every $m \in \Delta(x)$, the set $S_{\bar{\alpha}+m}$ is non-stationary. Let $\dot{X}_{\alpha+\omega \cdot(n+1)}$ be a $\mathbb{P}_{\alpha+\omega \cdot(n+1)}$-name for $X_{\alpha+\omega \cdot(n+1)}, \dot{\mathbb{K}}_{\alpha+\omega \cdot(n+1)}^{1}$ is a $\mathbb{P}_{\alpha+\omega \cdot(n+1)}$-name for $\mathbb{K}_{\alpha+\omega \cdot(n+1)}^{1}$.

- Let $Y_{\alpha+\omega \cdot(n+1)}$ be $\mathbb{K}_{\alpha+\omega \cdot(n+1)}^{1}$-generic filter over $L\left[G_{\alpha+\omega \cdot(n+1)}\right]$. In $L\left[Y_{\alpha+\omega \cdot(n+1)}\right]$ (note that the even part of $Y_{\alpha+\omega \cdot(n+1)}$ codes $X_{\alpha+\omega \cdot(n+1)}$ and so the generic $\left.G_{\alpha+\omega \cdot(n+1)}\right)$ let $\mathbb{K}_{\alpha+\omega \cdot(n+1)}^{2}=\mathcal{C}\left(Y_{\alpha+\omega \cdot(n+1)}\right)$ and let $R_{\alpha+\omega \cdot(n+1)}$ be the generic real added by $\mathbb{K}_{\alpha+\omega \cdot(n+1)}^{2}$. Let $\mathbb{Q}_{\alpha+\omega \cdot(n+1)}=\mathbb{K}_{\alpha+\omega \cdot(n+1)}^{1} *$ $\dot{\mathbb{K}}_{\alpha+\omega \cdot(n+1)}^{2}$.

In $L^{\mathbb{P}_{\alpha+\omega \cdot \omega}}$, let $u_{n}^{\alpha}=R_{\alpha+\omega \cdot n}$ for $n \in \omega$ (in particular $u_{0}^{\alpha}=x$.) Let $\vec{u}_{\alpha}=\left(u_{n}^{\alpha}\right)_{n \in \omega}$ and let

$$
g_{\alpha}=\bar{r}\left(F_{\alpha+\omega \cdot \omega}^{0}\left(u_{0}^{\alpha}\right), F_{\alpha+\omega \cdot \omega}^{1}\left(\left(u_{n}^{\alpha}\right)_{n \geq 1}\right)\right)
$$

(see Lemma 1). For every $\gamma \in[\alpha, \alpha+\omega \cdot \omega)$ let $O_{\gamma}=\left\{g_{\alpha}\right\}$. For $n \in \omega$, let $A_{\alpha+\omega \cdot n}=\alpha+\omega \cdot n+\omega \backslash \Delta\left(u_{n}^{\alpha}\right)$ and for $\gamma$ successor in $[\alpha, \alpha+\omega \cdot \omega)$, let $A_{\gamma}=\emptyset$.

Case B. Suppose $\alpha \in \operatorname{Lim}\left(\omega_{2}\right) \backslash \operatorname{Lim}^{\prime}\left(\omega_{2}\right)$, or $\alpha$ is a successor ordinal in $\omega_{2}$ which can not be presented in the form $\alpha^{\prime}+\omega \cdot n+m$ for some $\alpha^{\prime} \in \operatorname{Lim}^{\prime}\left(\omega_{2}\right), n, m \in \omega$. Then let $\dot{\mathbb{Q}}_{\alpha}$ be a $\mathbb{P}_{\alpha}$-name for the random real forcing. Let $O_{\alpha}=A_{\alpha}=\emptyset$.

With this the recursive construction of $\mathbb{P}_{\omega_{2}}$ is complete. Similarly to [1, Lemma 17], one can show that if $G$ is $\mathbb{P}_{\omega_{2}}$-generic and $\xi \in \bigcup_{\xi \in \omega_{2}} \dot{A}_{\xi}^{G}$, then $S_{\xi}$ is stationary in $L[G]$. We will refer to this fact as no accidental stationary kill. In $L^{\mathbb{P} \omega_{2}}$, let $O=\bigcup_{\alpha<\omega_{2}} O_{\alpha}, F^{0}=\bigcup_{\alpha \in \omega_{2}} F_{\alpha}^{0}, F^{1}=\bigcup_{\alpha \in \omega_{2}} F_{\alpha}^{1}$ and for $\vec{z}=\left(z_{n}\right)_{n \in \omega} \in\left(2^{\omega}\right)^{\omega}$ let $\mathcal{R}(\vec{z})=\bar{r}\left(F^{0}\left(z_{0}\right), F^{1}\left(\left(z_{n}\right)_{n \geq 1}\right)\right)$ (see Lemma 1).

Lemma 4.1 Let $G$ be $\mathbb{P}_{\omega_{2}}$-generic and let $g=\mathcal{R}(\vec{z}), \vec{z}=\left(z_{n}\right)_{n \in \omega}$. Then $g \in O$ if and only if for every countable suitable model $\mathcal{M}$ such that $g \in \mathcal{M}$, there is $\bar{\alpha}<\omega_{2}^{\mathcal{M}}$ such that for all $n \in \omega$ the set $S_{\alpha+\omega \cdot n+m}$ is non-stationary in $\left(L\left[z_{n+1}\right]\right)^{\mathcal{M}}$ for $m \in \Delta\left(z_{n}\right)$. 
Proof Suppose $g \in O$. Then $g=g_{\alpha}=\mathcal{R}\left(\vec{u}_{\alpha}\right)$ for some $\alpha$. Let $\mathcal{M}$ be a countable suitable model such that $g \in \mathcal{M}$. By definition of the function $\bar{r}$ we have that $F^{0}\left(u_{0}^{\alpha}\right)$ and $F^{1}\left(\left(u_{n}^{\alpha}\right)\right)_{n \geq 1}$ are elements of $\mathcal{M}$. Since $F^{0}, F^{1}$ are nicely definable, $\vec{u}_{\alpha} \in \mathcal{M}$, and so $Y_{\alpha+\omega \cdot n} \cap \omega_{1}^{\mathcal{M}} \in \mathcal{M}$ for all $n$. Thus $X_{\alpha+\omega \cdot n} \cap \omega_{1}^{\mathcal{M}}$ is also an element of $\mathcal{M}$. By definition of $\mathcal{L}\left(\phi_{\alpha+\omega \cdot n}^{n}\right)$, the set $X_{\alpha+\omega \cdot n} \cap \omega_{1}^{\mathcal{M}}$ codes a tuple $\left\langle W_{j}^{n}\right\rangle_{j \leq n}$ of subsets of $\omega_{1}$ such that $W_{n}^{n}$ is the $L$-least code of an ordinal $\alpha_{n}^{n}$ in $\omega_{2}$ and for $j<n$ the set $W_{j}^{n}$ is the $L$-least code for the largest limit ordinal $\alpha_{j}^{n}$ below $\alpha_{j+1}^{n}$. It remains to observe that $W_{j}^{n}=W_{j}^{m}$ for $j \leq n<m$ and so $\alpha_{0}^{n}$ does not depend on $n$. But then $\bar{\alpha}=\alpha_{0}^{n}$ is the desired ordinal.

Suppose that for every countable suitable model $\mathcal{M}$ such that $g \in \mathcal{M}$, there is $\bar{\alpha}<\omega_{2}^{\mathcal{M}}$ with the desired properties. By the Löwenheim-Skolem theorem, the same holds in $\mathbb{H}_{\Theta}^{\mathbb{P} \omega_{2}}$ for some large $\Theta$. Therefore there is $\alpha<\omega_{2}^{\mathcal{M}}$ such that for all $n \in \omega$, the set $S_{\alpha+\omega \cdot n+m}$ is non-stationary iff $m \in \Delta\left(z_{n}\right)$. Since there is no accidental stationary kill, $z_{n}=u_{n}^{\alpha}$ for all $n$, which implies that $g=\mathcal{R}\left(\vec{u}_{\alpha}\right)=g_{\alpha} \in O$.

Therefore $O$ indeed has a $\Pi_{2}^{1}$-definition. We will show that $O$ is maximal in $p_{c}\left(2^{\omega}\right)$. Suppose in $L^{\mathbb{P}_{\omega_{3}}}$ there is a code $f$ of a measure orthogonal to every measure in the family $\bar{O}=\left\{\mu_{g}: g \in O\right\}$. Choose $\alpha$ minimal in $\operatorname{Lim}^{\prime}\left(\omega_{2}\right)$ such that $f \in L\left[G_{\alpha}\right]$ and let $x=\left(F_{\alpha}^{0}\right)^{-1}(f)$. Since $F^{-1}\left(\sigma_{x}^{\alpha}\right)$ is unbounded, there is $\alpha^{\prime} \geq \alpha$ in $\operatorname{Lim}^{\prime}\left(\omega_{2}\right)$ such that $F\left(\alpha^{\prime}\right)=\sigma_{x}^{\alpha}\left(=\sigma_{x}^{\alpha^{\prime}}\right)$. But then $g_{\alpha^{\prime}}$ is a code of a measure equivalent to $\mu_{f}$, which is a contradiction. To obtain a $\Pi_{2}^{1}$-definable m.o. family in $L^{\mathbb{P} \omega_{3}}$, consider the union of $\bar{O}$ with the set of all point measures. Just as in [1] one can show that $<$ is indeed a $\Delta_{3}^{1}$-definable wellorder of the reals.

Since for every real $a \in L^{\mathbb{P} \omega_{3}}$ there is a random real over $L$, by Proposition 1 in $L^{\mathbb{P} \omega_{3}}$ there are no $\boldsymbol{\Sigma}_{2}^{1}$ m.o. families. The dominating number $\mathfrak{d}$ remains $\omega_{1}$ in $L^{\mathbb{P}_{\omega_{3}}}$, since the countable support iteration of $S$-proper $\omega_{\omega} \omega$-bounding posets is ${ }^{\omega} \omega$-bounding (see [1, Lemma 18] or [5]). This completes our proof of Theorem 2.

We conclude with some open questions.

Remark 4.2 In [3] the following question was raised:

Question 1 If there is a $\Pi_{1}^{1}$ m.o. family, are all reals constructible?

This is to our knowledge still unsolved. Törnquist has recently shown that the existence of a $\Sigma_{2}^{1}$ m.o. family implies the existence of a $\Pi_{1}^{1}$ m.o. family, and that the existence of $\Sigma_{2}^{1}$ mad family implies the existence of a $\Pi_{1}^{1} \operatorname{mad}$ family.

\section{References}

[1] V. Fischer, S. D. Friedman, Cardinal characteristics and projective wellorders, Annals of Pure and Applied Logic 161 (2010), 916-922; doi: 10.1016/j.apal.2009.11.003.

[2] V. Fischer, S. D. Friedman, L. Zdomskyy, Projective wellorders and mad families with large continuum, Annals of Pure and Applied Logic 162 (2011), 853-862; doi: 10.1016/j.apal.2011.04.001. 
[3] V. Fischer, A. Törnquist, A co-analytic maximal set of orthogonal measures, Journal of Symbolic Logic, 75 (2010), 1403-1414; doi: 10.2178/jsl/1286198154.

[4] S. D. Friedman, L. Zdomskyy, Projective mad families, Annals of Pure and Applied Logic 161 (2010), 1581-1587; doi: 10.1016/j.apal.2010.06.007.

[5] M. Goldstern, A taste of proper forcing, in Set Theory (Curaçao, 1995; Barcelona, 1996), 71-82, Kluwer Academic Publishers, Dordrecht, 1998.

[6] J. I. Ihoda, S. Shelah, $\Delta_{2}^{1}$-sets of reals, Annals of Pure and Applied Logic 42 (1989), 201-223; MR 998607(90f:03081); doi: 10.1016/0168-0072(89)90016-X.

[7] T., Set Theory, Springer Monographs in Mathematics, Springer-Verlag, Berlin, 2003.

[8] B. Kastermans, J. Steprāns, Y. Zhang, Analytic and coanalytic families of almost disjoint functions, Journal of Symbolic Logic 73 (2008), 1158-1172; doi: 10.2178/js1/1230396911.

[9] A. S. Kechris, Classical Descriptive Set Theory, Graduate Texts in Mathematics, 156, Springer-Verlag, 1995.

[10] A. Kechris, N. E. Sofronidis, A strong ergodic property of unary and self-adjoint operators, Ergodic Theory and Dynamical Systems 21 (2001), 1459-1479; doi: 10.1017/S0143385701001705.

[11] A. Miller, Infinite combinatorics and definability, Annals of Pure and Applied Logic 41 (1989), 179-203; doi: 10.1016/0168-0072(89)90013-4.

[12] A.R.D. Mathias, Happy Families, Annals of Mathematical Logic 12 (1977), 59-111.

[13] D. Preiss, J.Rataj, Maximal Sets of Orthogonal Measures are not Analytic Proceedings of the American Mathematical Society 93(3) (1985), 471-476; doi: 10.1090/S0002-9939-1985-0774005-7.

[14] D. Raghavan, Maximal almost disjoint families of functions, Fundamenta Mathematicae 204 (2009), 241-282; doi: 10.4064/fm204-3-3.

Kurt Gödel Research Center, University of Vienna, Währinger Strasse 25, A-1090 Vienna, Austria (Fischer \& Friedman) Department of Mathematics, University of Copenhagen, Universitetsparken 5, 2100 Copenhagen, Denmark (Törnquist) vfischer@logic.univie.ac.at, sdf@logic.univie.ac.at, asger@logic.univie.ac.at

Received: 28 June $2011 \quad$ Revised: 24 March 2012 